\title{
Holographic Imaging Reveals the Mechanism of Wall Entrapment in Swimming Bacteria
}

\author{
Silvio Bianchi, ${ }^{1,}{ }^{*}$ Filippo Saglimbeni, ${ }^{1}$ and Roberto Di Leonardo ${ }^{1,2}$ \\ ${ }^{1}$ CNR-NANOTEC, Soft and Living Matter Laboratory, Rome, I-00185 Roma, Italy \\ ${ }^{2}$ Dipartimento di Fisica, Università di Roma "Sapienza", Rome, I-00185, Italy \\ (Received 31 August 2016; revised manuscript received 18 November 2016; published 27 January 2017) \\ Self-propelled particles, both biological and synthetic, are stably trapped by walls and develop high \\ concentration peaks over bounding surfaces. In swimming bacteria, like E. coli, the physical mechanism \\ behind wall entrapment is an intricate mixture of hydrodynamic and steric interactions with a strongly \\ anisotropic character. The building of a clear physical picture of this phenomenon demands direct and full \\ three-dimensional experimental observations of individual wall entrapment events. Here, we demonstrate \\ that, by using a combination of three-axis holographic microscopy and optical tweezers, it is possible to \\ obtain volumetric reconstructions of individual $E$. coli cells that are sequentially released at a controlled \\ distance and angle from a flat solid wall. We find that hydrodynamic couplings can slow down the cell \\ before collision, but reorientation only occurs while the cell is in constant contact with the wall. In the \\ trapped state, all cells swim with the average body axis pointing into the surface. The amplitude of this \\ pitch angle is anticorrelated to the amplitude of wobbling, thus indicating that entrapment is dominated by \\ near-field couplings between the cell body and the wall. Our approach opens the way to three-dimensional \\ quantitative studies of a broad range of fast dynamical processes in motile bacteria and eukaryotic cells.
}

DOI: 10.1103/PhysRevX.7.011010

\section{INTRODUCTION}

When a self-propelled particle, moving with a persistent speed $v$, reaches an impenetrable boundary, it will stay on the boundary for a characteristic finite time $\tau$ before it reorients and returns back to the bulk. The stationary distribution of particles will then be characterized by a surface density component $\sigma$ such that the flux of bulk particles colliding with the surface is balanced by the rate of escapes from it. Using simple dimensional arguments [1,2], the flux of incoming particles scales as $\rho v$, with $\rho$ the bulk concentration, while the rate of escapes will scale as $\sigma / \tau$. By equating the two terms, we find that a finite surface density builds up scaling as $\rho v \tau$. Persistence is thus a sufficient condition for wall accumulation which does not require any further physical mechanism to keep the cell in contact with the boundary. Because of this, wall accumulation is observed in practically every theoretical model of active random walks with a finite persistence length [2-7]. When the boundary does not alter the reorientation dynamics of the particle, the escape time $\tau$ will simply scale with the bulk reorientation time so that $\sigma \propto \rho \ell$, where $\ell=v \tau$ is the persistence length of the active random walk in the

\footnotetext{
*silvio.bianchi.phys@gmail.com
}

Published by the American Physical Society under the terms of the Creative Commons Attribution 4.0 International license. Further distribution of this work must maintain attribution to the author(s) and the published article's title, journal citation, and DOI.
Subject Areas: Biological Physics, Fluid Dynamics, Optics

bulk [1]. In real swimmers, however, the presence of a physical boundary can strongly affect the propulsion mechanism by imposing no-slip boundary conditions on the solvent flow [8] or by hindering the motion of flagella $[9,10]$. These wall effects on motility can result in escape times $\tau$ that are much longer than the typical reorientation times in the bulk $[11,12]$. This results in surface-restricted trajectories that are much longer and much more stable than what would be expected by neglecting wall effects on motility. We call this effect wall entrapment. Swimming bacteria, like E. coli, display a pronounced wall accumulation effect [13-15]. In addition to being an interesting dynamical problem on its own, cell-wall interactions have a strong biological relevance in the first stages of biofilm formation [16-18]. Moreover, understanding cell-wall interactions is also important in designing wall geometries that can be used to direct bacteria inside microfluidic devices $[19,20]$ or exploit bacteria as tiny propellers in microstructures [21,22]. Despite these broad implications of wall accumulation in E. coli, in current literature it is still under debate whether it is a purely statistical effect arising from steric interactions [23] or rather a wall-entrapment phenomenon of hydrodynamic origin [24]. On the theoretical side, it has been noted that hydrodynamic couplings to the wall can provide a wall-entrapment mechanism already in the far field [24]. Using boundary-element methods, direct numerical simulations of rigid swimmers moving in close proximity to a no-slip wall have found the existence of stable trajectories with the cell swimming at a finite distance from the wall in a "nose-up" configuration, 
i.e., with the cell long axis pointing away from the surface by a small tilt angle [25-28]. More refined mesoscale hydrodynamic simulations of mechano-elastic cell models, including thermal noise and steric interactions, also find stable trajectories with a cell axis that is again almost perfectly aligned with the surface [29]. Similar simulations of swarmer-type cells, having a longer shape and a random distribution of flagella over the surface, show instead that a complex mixture of near-field hydrodynamics and steric repulsion leads to stable trapped states with cells that are preferentially oriented towards the wall [30]. On the experimental side, the analysis of accumulation profiles of bacteria can be interpreted equally well with stochastic models that either include hydrodynamics in the far-field approximation [24] or disregard it completely [23]. A clear experimental demonstration and description of a wallentrapment effect necessarily requires a three-dimensional (3D) imaging technique, allowing the systematic analysis of both positions and orientations.

Here, we demonstrate that, by using a combination of three-axis holographic microscopy and optical tweezers, it is possible to reconstruct the full $3 \mathrm{D}$ entrapment dynamics of smooth swimming $E$. coli cells that are sequentially released at a controlled distance and angle from a flat solid wall. We analyzed about 300 individual entrapment events where we could resolve the three main stages of wall entrapment (approach, alignment, and surface swimming). For each of these stages, we could quantitatively gauge the relative importance of the involved physical mechanisms. For instance, we found that cell reorientation starts at contact, where steric interactions are dominant. Steric forces alone, however, are not sufficient to account for stable surface swimming, which always occurs with the cell pointing into the surface ("nose down"). We also found that wobbling, i.e., a precession motion of the cell-body axis around the swimming direction, plays an important role in determining both swimming pitch angle and distance from the wall.

\section{3D VISUALIZATION OF COLLISION EVENTS STARTING FROM CONTROLLED INITIAL CONFIGURATIONS}

Even if precise 3D tracking of bacteria positions is possible [13-15,23,31-33], obtaining volumetric images of swimming cells is a rather challenging task. Bacteria like $E$. coli have a body length of approximately $2 \mu \mathrm{m}$ that is propelled by a spinning bundle of helical flagella at an average speed of about $20 \mu \mathrm{m} / \mathrm{s}$ [34,35]. A reasonable time resolution for tracking would be such that the cell body is displaced by $1 / 10$ of its size between frames, giving a required frame rate of about $100 \mathrm{fps}$. This requirement has to be combined with a submicron spatial resolution in both transverse and axial directions to allow precise tracking of both position and orientation of the swimming cell. A typical wall-trapping event takes place in a three-dimensional field of view measuring about $50 \times 50 \times 10 \mu \mathrm{m}^{3}$. The combination of all these requirements rules out the possibility of using microscopy techniques relying on a mechanical scan of the focal plane, i.e., confocal microscopy since, apart from recent advances where $512 \times 512$-pixel plane sections can be acquired at up to $200 \mathrm{~Hz}$ [36], most of the solutions offer video-rate frame rates for a single plane [37]. Here, we use a novel three-axis approach to in-line holographic microscopy [38,39], where volumetric images can be obtained with 0.4 and $0.8 \mu \mathrm{m}$ of, respectively, lateral and axial resolution at a frame rate that is mainly limited by the camera and that was $100 \mathrm{fps}$ in our case. The working principle of our technique is summarized in Fig. 1. Although in-line holographic microscopy has been used previously for center-of-mass tracking of microparticles $[40,41]$, or indirect 3D shape reconstruction through fitting procedures [42], our threeaxis implementation provides an unprecedented axial resolution that allows for direct 3D imaging of the prolate cell body. A quantitative approach requires, in addition to a suitable 3D microscopy technique, the possibility of generating a large number of collision events starting from controlled initial configurations. Optical trapping provides an ideal tool in this respect $[43,44]$. The cell body of $E$. coli can be stably trapped in three dimensions and with limited photodamage, using optical traps in the near infrared [39,45-47]. The elongated cell body also experiences a restoring torque that aligns the body axis along the trapping beam axis. This last axis can be easily tilted by deliberately underfilling the objective entrance pupil on one side. More precise control of cell orientation can be achieved using two holographic traps to grab the cell body at the two poles. Individual cells were trapped, oriented, and released from a fixed distance of about $10 \mu \mathrm{m}$ from the top glass wall of the sample chamber. A movie showing a sequence of collision events can be found in Ref. [48]. We acquired a total of 278 bacteria tracks, with initial pitch angles ranging from 50 to $90^{\circ}$.

Figure 2(a) shows a wall-entrapment event from a distant viewpoint that highlights the phenomenon of circular clockwise swimming over a no-slip interface [49]. A closer look from a lateral viewpoint is reported in Fig. 2(b), showing a sequence of superimposed volumetric reconstructions viewed from a direction that is orthogonal to the surface normal. The time evolution of height $z$ and cell pitch angle $\theta$ with respect to the wall surface are plotted in the inset [Fig. 2(c)] for the same cell. As the cell swims towards the surface, the distance $z$ between cell-body center and interface drops down linearly up to $t=0.75 \mathrm{~s}$, where a sharp change in slope is observed. Eventually, the distance reaches a stationary value, for $t>1.25 \mathrm{~s}$, where the cell has been trapped by the wall and swimming is restricted to the wall surface. On the same graph, we report the time evolution of the cell pitch angle $\theta$, where two components can be distinguished in the signal: a fast (about $8 \mathrm{~Hz}$ ) precession of the body (wobbling $[39,47]$ ) and a slower 


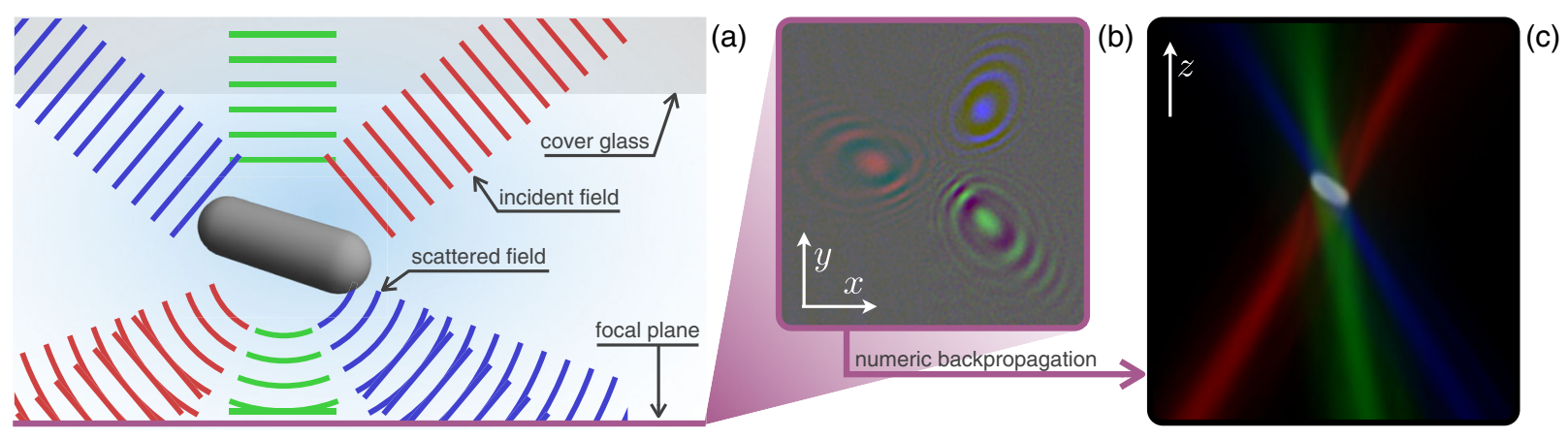

FIG. 1. Working principle of three-axis holographic microscopy. (a) The sample is illuminated by three partially coherent beams having different colors and directions. (b) The resulting hologram arising from the interference between scattered and unscattered light is acquired by a RGB camera. (c) A volumetric image of the sample is obtained as the overlap of three independent reconstructions obtained by numerically backpropagating the holograms (see also Ref. [48]).

alignment of the precession axis from an impact angle of approximately $60^{\circ}$ to a steady positive angle of about $10^{\circ}$ with respect to the wall surface. Three regions are again clearly distinguishable. Before $t=0.75 \mathrm{~s}$, the angle $\theta$ wobbles around a roughly constant value. Most of the reorientation occurs between $0.75 \mathrm{~s}$ and $1.25 \mathrm{~s}$, while for $t>1.25 \mathrm{~s}$, the angle oscillates around a stationary and small positive value. This qualitative subdivision in three stages emerges quite clearly in all trajectories, although quantitative differences are present because of the high variability of cell length, speed, and wobbling amplitude. The condition for which the cell spherocylindrical body and coverslip are in contact is given by $z=$ $(a-b) \sin (\theta)+b$ (where $2 a$ and $2 b$ are, respectively, the cell-body length and thickness). We can look at all the tracks at once, plotting $\theta$ versus the normalized vertical coordinate $(z-b) /(a-b)$, as shown in Fig. 3(a). In this way, the contact condition, plotted as a black dashed line, is independent from $a$ and $b$; also, the variability due to different cell speeds is eliminated.

\section{A. Stage 1: Approach}

We begin by discussing the first stage, the approach, defined by the condition $z-b / a-b>\sin \theta$, where the cell can interact with the surface only through the fluid and not by direct-contact interactions. In Fig. 3(a), we show, with gray lines, the raw $\theta$ vs $(z-b) /(a-b)$ curves. As already discussed, because of a high and variable degree of wobbling, traces form a thick cloud, but an overall scenario is still clearly visible. Three representative cell trajectories are highlighted in color. The blue and red traces correspond to bacteria with small wobbling angles, having high (about $90^{\circ}$ ) and low (about $60^{\circ}$ ) start angles, respectively. The green line represents a strongly wobbling cell that approaches the wall at an intermediate impact angle. In all three cases, the impact angle $\theta$ does not show a significant reduction from the large distance value to the collision line [dashed line in Fig. 3(a)], meaning that there is no visible "roundout" (parallel alignment) solely due to hydrodynamic couplings to the wall. On the contrary, deterministic simulations of an E. coli-like dipole swimmer in the
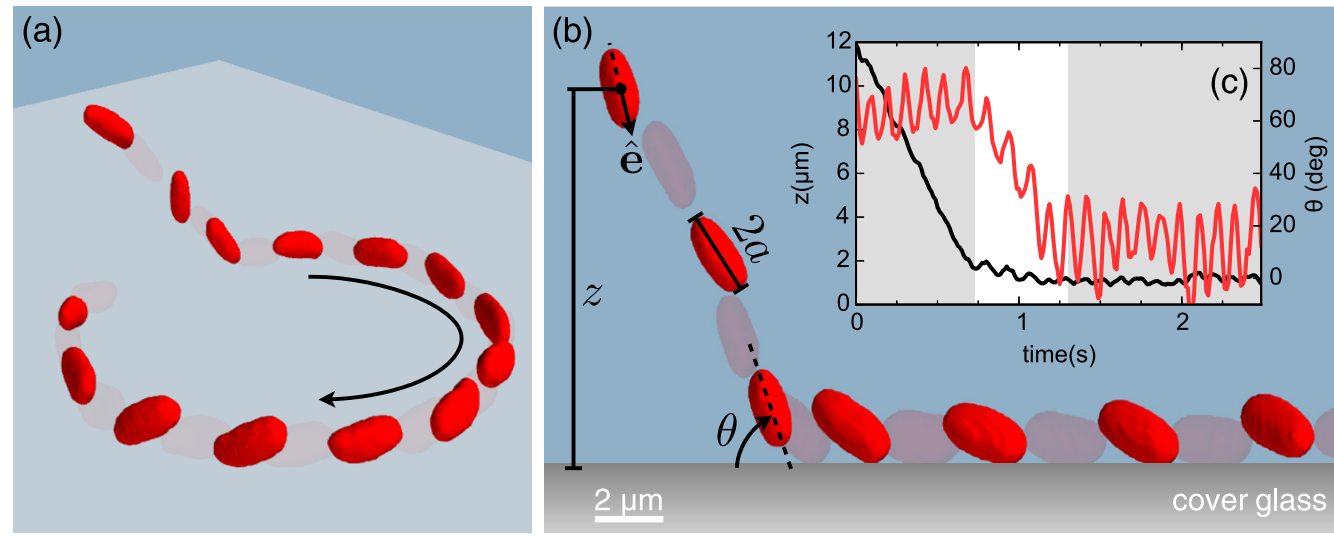

FIG. 2. (a) Sequence of volumetric reconstructions of swimming cells during a wall-entrapment event. (b) A close and lateral view of another cell colliding with the wall. Time intervals between reconstructions are $0.2 \mathrm{~s}$ in both figures. (c) For the same cell as in (b), the wall distance is plotted as a black line, while the red line plots the angle of the cell-body axis. In both curves, three stages can be identified: approach to the wall, reorientation, and surface swimming. The grey shaded areas help to visualize these three stages. 


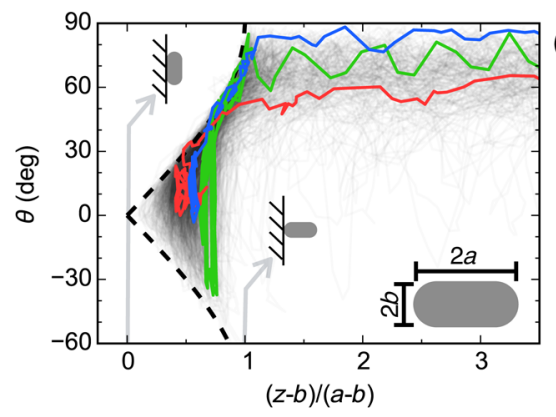

(a)

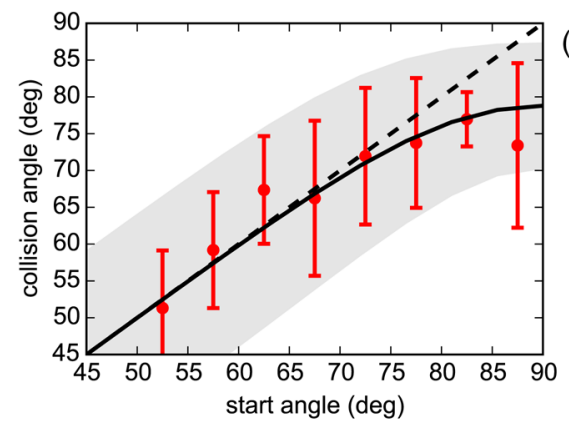

(c)

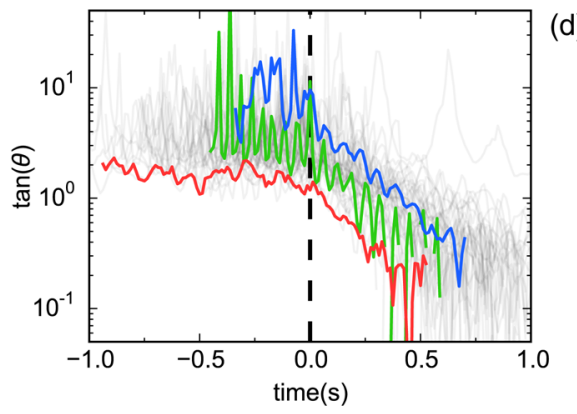

(b)

(d)

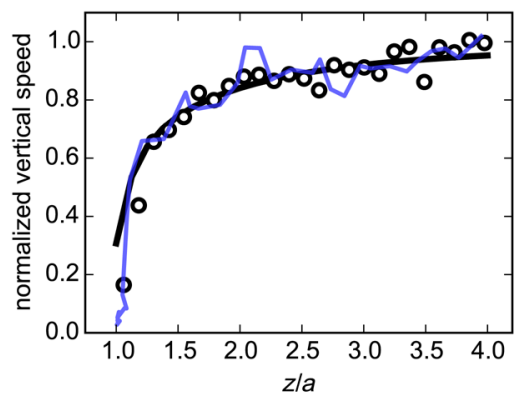

FIG. 3. (a) Gray lines plot the body pitch $\theta$ as a function of the normalized distance $(z-b) /(a-b)$. Blue, green, and red lines refer to bacteria approaching the wall with, respectively, high, intermediate, and low impact angles. The black dashed line represents the contact condition $[(z-b) /(a-b)=|\sin \theta|]$. (b) Collision angle at first impact plotted versus the corresponding starting value (red dots). Error bars represent $+/-$, the standard deviation over cells with a start angle falling in the same $5^{\circ}$ bin interval. The solid black line is the theoretical prediction for cells reorienting with a purely diffusive motion $\left(D_{r}=0.06 \mathrm{rad}^{2} / \mathrm{s}\right)$, while the shaded area represents the corresponding standard deviation. (c) The blue line plots the vertical component of the velocity as a function of the cell-wall distance for a bacterium approaching the glass wall almost perpendicularly $\left(\theta \approx 90^{\circ}\right)$. The velocity has been normalized to its bulk value, while the cell-wall distance $z$ is divided by the cell-body half-length $a$. The same quantity, averaged over all bacteria having an angle $\theta>70^{\circ}$, is shown as a blue line. The black line plots a theoretical prediction that only accounts for an increase of the cell-body drag due to the presence of the wall [see Eq. (1)]. (d) Time evolution of $\tan \theta$ during reorientation. Color coding is the same as in (b). For each curve, the time origin has been shifted so that the cell first hits the wall at time $t=0$.

presence of an infinite no-slip surface indicate that the cell should reorient by approximately $10^{\circ}$ just because of hydrodynamic interactions [11]. To better quantify the amount of reorientation arising from purely hydrodynamic couplings, we compared the initial and final orientations of the average cell-body axis (over a wobbling period) during the approach stage, starting from trap release and ending at first wall contact. We group cells by start angle, using $5^{\circ}$ bin intervals, and compute the mean and standard deviation of the collision angle. Results are shown in Fig. 3(b). Collision angles show no systematic reduction from the corresponding start value up to about $75^{\circ}$. The reduction observed for close to orthogonal orientations is compatible with a purely diffusive motion of the cell axis with a diffusion coefficient estimated to be $D_{r}=$ $0.06 \mathrm{rad}^{2} / \mathrm{s}$ (solid black line), which is also close to the value reported in Ref. [11]. If any hydrodynamic contribution to cell reorientation is present, it has to be smaller than expected and hidden by a strong diffusive dynamics. Conversely, hydrodynamic effects become clearly visible when we look at the vertical speed of approach as a function of cell height $z$, as reported in Fig. 3(c). The blue line refers to the same weakly wobbling cell in Fig. 3(a), while open circles represent the average speed, for all trajectories having a start angle larger than $70^{\circ}$. A marked reduction in vertical speed is observed that is qualitatively consistent with the far-field picture of bacteria as dipolar "pushers" that slow down in approaching the wall because of the backflow generated by image singularities at a noslip boundary [8]. However, a dipolar representation is not enough to quantitatively account for the observed speed reduction unless we move the dipole origin on a point that is unphysically closer to the cell-body pole facing the wall. One possibility could be that of considering higher-order singularities, but a breakdown of far-field predictions is expected for the distance range examined here [8]. Therefore, we choose to follow Ref. [50], and since during approach the flagellar bundle will always point away from the surface and cells will impact the wall with the cell body, we only consider body-wall hydrodynamic coupling and neglect bundle-wall interactions. We numerically computed the axial translational resistance $A_{b}(z)$ of a spherocylinder aligned along the wall normal and for different heights $z$. The vertical speed is then obtained by assuming that both flagellar thrust and resistance $A_{f}^{0}$ are constant and that the resistance of the full cell can be obtained by simply adding the bundle and body resistances: 


$$
\frac{v_{z}(z)}{v_{z}^{0}}=\frac{A_{b}^{0}+A_{f}^{0}}{A_{b}(z)+A_{f}^{0}} .
$$

Using Lighthill's slender-body theory [51] with bundle parameters as in Ref. [52], we estimate $A_{f}^{0}=0.011 \mathrm{pNs} / \mu \mathrm{m}$. The cell-body dimensions were set to the average measured length and thickness in our samples. Our results, reported as a black solid line in Fig. 3(c), show an excellent agreement with experimental data with no fitting parameters involved.

\section{B. Stage 2: Reorientation}

The approach stage is followed by a markedly different stage during which most of the reorientation occurs with the cell body being in constant contact with the surface. During reorientation, all trajectories in the $\theta, z$ space of Fig. 3(a) closely follow the dashed line marking the contact condition $z=(a-b) \sin \theta+\mathrm{b}$. A complete and accurate description of this second stage would require a mixture of near-field hydrodynamic interactions and direct contact forces, which is beyond the scope of the present paper. We simply notice here that from a purely hydrodynamic calculation based on far-field flows, the cell is expected to reorient with an angular velocity whose leading term is given by $d \theta / d t \propto-\sin \theta \cos \theta$ [8] or, equivalently, $d \tan \theta / d t \propto-\tan \theta$. A very similar form is obtained if we neglect hydrodynamic couplings and only consider steric interactions with the wall. Based on these two observations, a similar behavior for $d \tan \theta / d t$ is expected to be found even in a more complete treatment of reorientation dynamics close to contact. If this is correct, then we should expect to see that the tangent of the angle $\theta$ relaxes exponentially. Figure 3(d) reports the time evolution of $\tan \theta$ for all traces that display a clear reorientation phase; highlighted in color are the same three tracks shown in Fig. 3(a). A clear exponential decay is visible, especially in the blue track corresponding to a weakly wobbling cell arriving at almost normal incidence. All tracks have been shifted along the time axis to have time $t=0$ corresponding to the collision time, that is, the time when the cellsurface gap falls below a fixed threshold or, in other words, when the corresponding track in Fig. 3(a) first hits the dashed contact line. The fact that the exponential decay starts right after $t=0$ indicates that contact interactions are the primary driving force for the exponential reorientation. Far-field hydrodynamics would also give rise to an exponential decay, but if it were the main mechanism here, we would expect to see an exponential decay starting before $t=0$.

\section{Stage 3: Surface swimming}

If hydrodynamic effects were completely negligible, steric forces alone would reorient the cell parallel to the surface and the angle $\theta$ would decay all the way down to zero. On the contrary, tracks in Fig. 3(a) leave the contact line before ending at zero, and the angle $\theta$ starts to oscillate around a finite positive value. For each cell, we compute the average pitch angle $\bar{\theta}$ as the angle formed by the timeaveraged cell-body axis and the wall surface. The wobbling angle $\theta_{w}$ is defined as the angle formed by the body axis and its precession axis obtained by averaging over a wobbling period. In Fig. 4, we plot $\bar{\theta}$ and $\theta_{w}$ for 278 bacteria in the surface swimming stage. The magnitude of $\bar{\theta}$ and $\theta_{w}$ can vary a lot among cells. In particular, we find a mean $\bar{\theta}$ of $10^{\circ}$ with a standard deviation of $7^{\circ}$, while the mean and standard deviation of $\theta_{w}$ are, respectively, $20^{\circ}$ and $8^{\circ}$. We first point out that the vast majority of cells swim "nose down," with a positive $\bar{\theta}$ and therefore with the flagellar bundle that constantly pushes the cell body down onto the surface. This possibility was first proposed in Ref. [31] on the basis that elongated bodies translating over a surface are subject to two opposing hydrodynamic torques that might be balanced at a finite positive angle. A qualitatively similar result can also be found through a far-field analysis, including multipolar order higher than the lowest dipole term [8]. A first experimental indication that a cell might swim at a positive angle was given in Ref. [50], although standard epifluorescence microscopy only allowed one to study bacteria swimming at the bottom wedge of a vertical wall. For the first time here, having access to 3D volumetric reconstructions, we can observe $E$. coli cells swimming over a large flat surface from a lateral viewpoint. We find that the mean pitch angle is $10^{\circ}$, with peak values that can exceed $30^{\circ}$. However, it is not at all obvious that the lifting torque that brings the cells to such a high pitch angle is entirely hydrodynamic in origin. Full hydrodynamic simulations using boundary-element methods have been used to solve Stokes equations for a prolate cell body propelled by a rigid helical propeller. All these studies [25-28] consistently find a small and negative (nose-up) angle, which is in striking contrast to our data. A more refined model, which simulates individual flagella in the bundle explicitly using a mechano-elastic approach, finds again a practically zero average pitch angle, although large fluctuations are present [29]. In this previous model, four flagella are symmetrically arranged in close proximity to one of the cell poles, and a bundle forms along the same direction as the body long axis. More recently, similar simulations have been performed for a swarmer-type model, having many flagella randomly distributed over the cell surface [30]. In this last case, a bundle forms with a main axis that is tilted with respect to the cell-body long axis by an angle that is comparable to what was observed in our experiments. These model cells also display a high degree of wobbling and are found to swim at relatively large positive pitch angles $\left(2^{\circ}-10^{\circ}\right)$. These simulations seem to suggest that a positive correlation is to be expected between the wobbling angle and the pitch angle. On the contrary, experimental pitch angles are anticorrelated with 


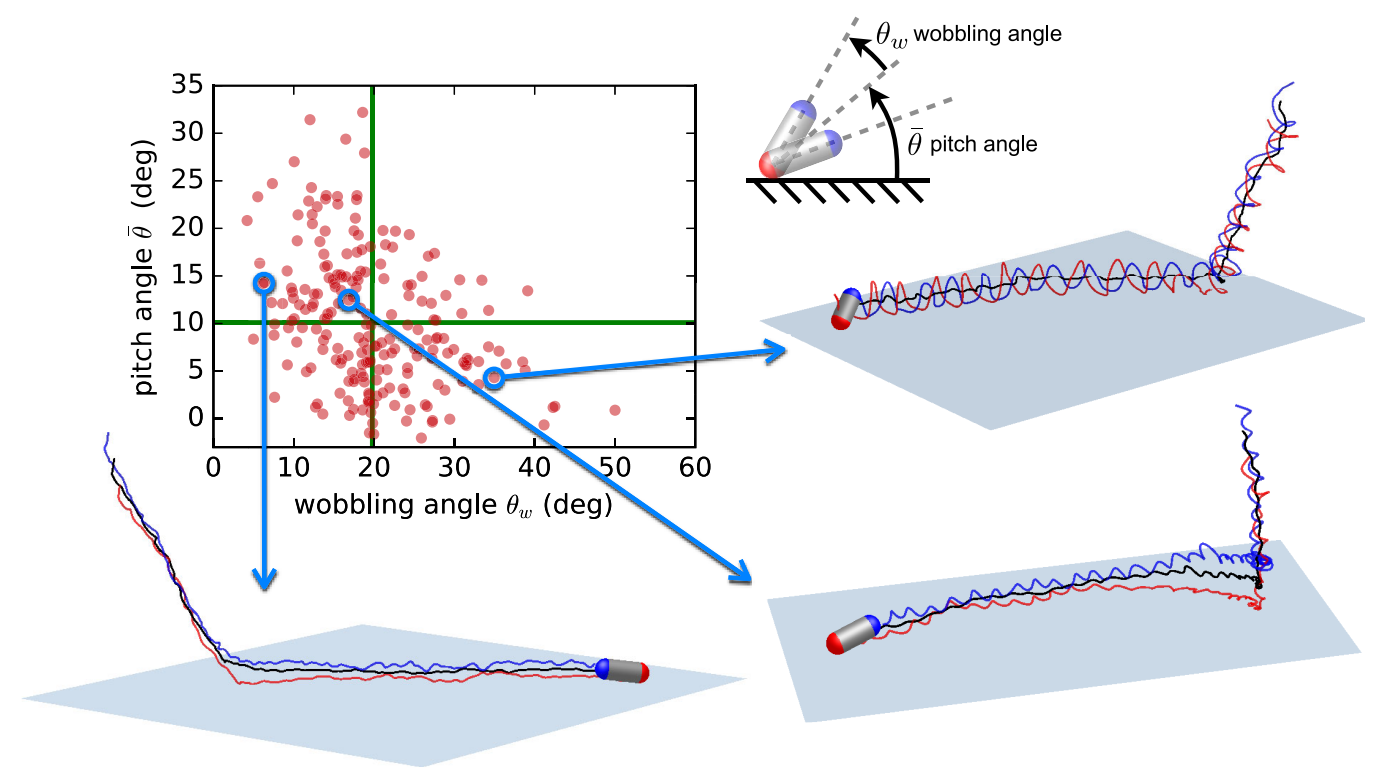

FIG. 4. Scatter plot of the time-averaged pitch angle $\bar{\theta}$ and the wobbling angle $\theta_{w}$ for cells that are swimming close to the surface. Green vertical and horizontal lines represent the mean over all the analyzed bacteria. An anticorrelation between $\bar{\theta}$ and $\theta_{w}$ is visible. Three selected cells' trajectories with small, average, and large wobbling are also shown. The black line represents the trajectory of the center of mass, while blue and red lines represent, respectively, the back and front poles.

the wobbling angle: Weakly wobbling cells swim at large inclination angles, while strong wobblers tend to swim with an average orientation that is more parallel to the surface (Fig. 4). It is important to remark here that neither hydrodynamics nor anisotropic steric interactions with the wall are necessary to observe an average polarization of swimmers that points towards the wall [53]. As already described in the Introduction, even purely spherical particles having no hydrodynamic interactions, but moving with a persistent speed, they will accumulate over a confining wall and remain in contact with the wall as long as the propulsion speed points towards it. This is already enough to generate a positive polarization towards the wall. The same particles, however, will reorient freely and leave the wall on a time scale that is given by the typical reorientation time in the bulk. What we observe here is instead a stable polarization towards the wall that persists for the entire observation time and cannot be accounted for without introducing some extra wall coupling mechanism that constrains $\bar{\theta}$ in the neighborhood of a positive value. Although hydrodynamic couplings can result in such nosedown configurations $[8,31,50]$, we hope that forthcoming accurate simulations will clarify whether hydrodynamics alone is sufficient to quantitatively reproduce our experimental findings.

As opposed to the reorientation phase, in the surface swimming stage, the cell is not in constant contact with the surface, the coordinates $(z, \theta)$ leave the contact line, and the cell wobbles at an approximately constant value for $z$ [see Fig. 3(a)]. To better highlight this 3D character of surface swimming, we report in Fig. 4 three selected trajectories with small, average, and large wobbling angle. In all three trajectories, the center of mass moves on a smooth path (black line), while the front and back poles of the cell, respectively in red and blue, trace helicoidal paths. This observation can be explained as follows. During reorientation, $\theta$ is sufficiently large, so there is a substantial vertical component of thrust that guarantees constant contact between the cell and the wall. After reorientation, the cell is almost parallel to the surface, and vertical thrust is not large enough to maintain the cell in contact with the wall when the front pole lifts up from the surface because of wobbling. The magnitude of the pitch angle is expected to increase when the front pole is closer to the surface and large viscous stresses in the gap develop a torque that tends to tilt the cell nose down $[8,50]$. Moreover, cells that wobble more will have a front pole at a higher average distance from the surface compared to weakly wobbling cells. These arguments provide a possible explanation for the fact that cells that wobble more will have a smaller pitch angle, as shown by the data in Fig. 4.

\section{DISCUSSION}

We have shown that our three-axis implementation of inline digital holographic microscopy provides the combination of high speed and good axial resolution that is required to observe the full three-dimensional dynamics of rod-shaped swimming bacteria approaching a solid wall. Through an extensive and quantitative analysis of the recorded volumetric movies, we could assess the 
relative importance of the main physical mechanisms involved in the wall-entrapment phenomenon. We conclude that, although hydrodynamic couplings slow down cells as they approach the wall, it is mainly through steric forces at contact that the average cell axis reorients in a direction that is parallel to the wall plane. However, the reorientation is not complete, and near-field hydrodynamic couplings between the cell body and the wall maintain the body axis at an inclined direction, with the cell front pole pointing into the surface. This mechanism is essential for stable trapping, while steric forces alone would not prevent escape from the wall because of orientational diffusion. The available full 3D information also reveals that the amount of cell wobbling plays an important role in determining mean pitch angle and distance from the wall in the trapped state. We still need to understand the physical origin of the force that lifts the back of the cell and produces a positive average pitch angle. Although pure hydrodynamic arguments seem to provide a justification, accurate numerical simulations fail to reproduce experimental data unless individual flagella are included explicitly and the cell is treated as a complex dynamical body. If forthcoming full-hydrodynamic simulations with purely repulsive wall interactions fail to quantitatively reproduce our findings, then a more refined description of the cell-wall interaction will be required. In this respect, our approach could provide further insights by studying mutant strains with different surface properties (i.e., pili-deficient mutants) or surfaces, like liquid-air interfaces, that provide different boundary conditions. Prokaryotic flagella are too thin and do not scatter enough light to be visualized in 3D with a holographic technique like ours. In this case, integrating fluorescence imaging in our setup could at least provide important information on the bundle morphology and conformation [52]. On the contrary, thicker eukaryotic flagella could be directly imaged in 3D with our method, which could provide an important tool to investigate a broad range of intrinsically three-dimensional and fast dynamical phenomena in motile cells like spermatozoa or flagellated algae.

\section{ACKNOWLEDGMENTS}

The research leading to these results has received funding from the European Research Council under the European Unions Seventh Framework Programme (FP7/2007-2013)/ERC Grant Agreement No. 307940.

\section{APPENDIX: MATERIALS AND METHODS}

\section{Cell growth}

E. coli cells (smooth swimming strain HCB437 [54]) were streaked on a Petri dish containing $1.5 \%$ of agar and tryptone broth $(1 \%$ tryptone and $0.5 \% \mathrm{NaCl})$. A single colony was inoculated into tryptone broth and grown overnight at $33{ }^{\circ} \mathrm{C}$ with $200 \mathrm{rpm}$ rotation. The saturated culture was then diluted $1: 100(50 \mu \mathrm{l}$ in $5 \mathrm{ml})$ into fresh medium and grown for $4 \mathrm{~h}$ at $33{ }^{\circ} \mathrm{C}$ with $200 \mathrm{rpm}$ rotation. Bacterial cells were then harvested from culture media by centrifugation at $8000 \mathrm{rpm}$ for $2 \mathrm{~min}$ at room temperature. The pellet was resuspended by gently mixing in trapping buffer composed of potassium phosphate $100 \mathrm{mM}\left(\mathrm{K}_{2} \mathrm{HPO}_{4}, \mathrm{KH}_{2} \mathrm{PO}_{4}, \mathrm{pH}\right.$ 7.0), $100 \mathrm{mM}$ EDTA, $2 \%(\mathrm{wt} / \mathrm{vol})$ glucose, $0.2 \%$ Tween 20 , and an oxygen scavenging system $[45,47]$. The cells were washed three times to replace growth medium with trapping buffer. Trapping buffer sustains bacteria motility and reduces the oxidative damage to the cell induced by optical trapping $[45,46]$. We used a diluted solution of bacteria for filling a $50 \mu \mathrm{m}$ thick chamber made by a glass slide and a cover glass glued together with a UV photoresist. Both the glass slides were treated with a solution of sulfuric acid and a glass-cleaning compound (NOCHROMIX) before washing with purified water.

\section{Optical setup}

The sample is illuminated by three partially coherent LED beams (with wavelengths 454, 525, and $637 \mathrm{~nm}$ ) forming a $45^{\circ}$ angle with the optical axis and equally spaced along the azimuthal coordinate. Three independent holograms, produced by the interference between illumination and scattered light, are acquired by the three channels of a RGB camera in a single snapshot. Using numerical backpropagation, we reconstruct the field in a 3D region of about $50 \times 50 \times 20 \mu \mathrm{m}^{3}$. Our setup is also equipped with a holographic trapping system based on an infrared laser $(\lambda=1064 \mathrm{~nm})$ and a LCOS spatial light modulator. A more detailed description of the setup can be found in Refs. [38,39].

\section{Analysis of volumetric images}

Using the first two spatial moments of the 3D intensity distributions, we obtain the center-of-mass coordinates $x, y$, $z$, as well as the three components of the body axis $\hat{\mathbf{e}}=$ $\left(e_{x}, e_{y}, e_{z}\right)$ as illustrated in Fig. 2(b). Cell lengths $2 a$ are directly obtained from volumetric images, while we assume a fixed thickness $2 b$ for all the cells [55]. Using fluorescence microscopy, we found an average thickness of $b=0.4 \mu \mathrm{m}$, with cell-to-cell variations that are small compared to our resolution. We do not see the coverslip directly, so the vertical position of the wall $z_{0}$ has to be determined for each track by looking at the cell trajectory. We compute $z_{0}$ as the minimum of $z-(a-b)|\sin (\theta)|-b$, representing the lowest point on the surface of a spherocylindrical body of semiaxes $a$ and $b$. For several tracks, we could confirm the validity of our procedure by comparing the obtained values of $z_{0}$ with the position of small particles that are occasionally found stuck on the wall surface. 
[1] J. Elgeti and G. Gompper, Run-and-Tumble Dynamics of Self-Propelled Particles in Confinement, Europhys. Lett. 109, 58003 (2015).

[2] B. Ezhilan, R. Alonso-Matilla, and D. Saintillan, On the Distribution and Swim Pressure of Run-and-Tumble Particles in Confinement, J. Fluid Mech. 781, R4 (2015).

[3] J. Elgeti and G. Gompper, Self-Propelled Rods Near Surfaces, Europhys. Lett. 85, 38002 (2009).

[4] J. Tailleur and M. E. Cates, Sedimentation, Trapping, and Rectification of Dilute Bacteria, Europhys. Lett. 86, 60002 (2009).

[5] W. Yan and J. F. Brady, The Force on a Boundary in Active Matter, J. Fluid Mech. 785, R1 (2015).

[6] C. Maggi, U. M. B. Marconi, N. Gnan, and R. Di Leonardo, Multidimensional Stationary Probability Distribution for Interacting Active Particles, Sci. Rep. 5, 10742 (2015).

[7] C. Bechinger, R. Di Leonardo, H. Löwen, C. Reichhardt, G. Volpe, and G. Volpe, Active Brownian Particles in Complex and Crowded Environments, Rev. Mod. Phys. 88, 045006 (2016).

[8] S. E. Spagnolie and E. Lauga, Hydrodynamics of SelfPropulsion Near a Boundary: Predictions and Accuracy of Far-Field Approximations, J. Fluid Mech. 700, 105 (2012).

[9] V. Kantsler, J. Dunkel, M. Polin, and R. E. Goldstein, Ciliary Contact Interactions Dominate Surface Scattering of Swimming Eukaryotes, Proc. Natl. Acad. Sci. U.S.A. 110, 1187 (2013).

[10] M. Contino, E. Lushi, I. Tuval, V. Kantsler, and M. Polin, Microalgae Scatter Off Solid Surfaces by Hydrodynamic and Contact Forces, Phys. Rev. Lett. 115, 258102 (2015).

[11] K. Drescher, J. Dunkel, L. H. Cisneros, S. Ganguly, and R. E. Goldstein, Fluid Dynamics and Noise in Bacterial Cell-Cell and Cell-Surface Scattering, Proc. Natl. Acad. Sci. U.S.A. 108, 10940 (2011).

[12] K. Schaar, A. Zöttl, and H. Stark, Detention Times of Microswimmers Close to Surfaces: Influence of Hydrodynamic Interactions and Noise, Phys. Rev. Lett. 115, 038101 (2015).

[13] P. D. Frymier, R. M. Ford, H. C. Berg, and P. T. Cummings, Three-Dimensional Tracking of Motile Bacteria Near a Solid Planar Surface, Proc. Natl. Acad. Sci. U.S.A. 92, 6195 (1995).

[14] M. A. Vigeant and R. M. Ford, Interactions between Motile Escherichia Coli and Glass in Media with Various Ionic Strengths, as Observed with a Three-Dimensional-Tracking Microscope, Appl. Environ. Microbiol. 63, 3474 (1997).

[15] P. D. Frymier and R. M. Ford, Analysis of Bacterial Swimming Speed Approaching a Solid-Liquid Interface, AIChE J. 43, 1341 (1997).

[16] L. A. Pratt and R. Kolter, Genetic Analysis of Escherichia Coli Biofilm Formation: Roles of Flagella, Motility, Chemotaxis and Type I Pili, Mol. Microbiol. 30, 285 (1998).

[17] T. K. Wood, A. F. González Barrios, M. Herzberg, and J. Lee, Motility Influences Biofilm Architecture in Escherichia Coli, Appl. Microbiol. Bioltech. 72, 361 (2006).

[18] J. C. Conrad, Physics of Bacterial Near-Surface Motility Using Flagella and Type IV Pili: Implications for Biofilm Formation, Res. Microbiol. 163, 619 (2012).

[19] W. R. DiLuzio, L. Turner, M. Mayer, P. Garstecki, D. B. Weibel, H. C. Berg, and G. M. Whitesides, Escherichia Coli
Swim on the Right-Hand Side, Nature (London) 435, 1271 (2005).

[20] P. Galajda, J. Keymer, P. Chaikin, and R. Austin, A Wall of Funnels Concentrates Swimming Bacteria, J. Bacteriol. 189, 8704 (2007).

[21] R. Di Leonardo, L. Angelani, D. DellArciprete, Giancarlo Ruocco, V. Iebba, S. Schippa, M. P. Conte, F. Mecarini, F. De Angelis, and E. Di Fabrizio, Bacterial Ratchet Motors, Proc. Natl. Acad. Sci. U.S.A. 107, 9541 (2010).

[22] A. Sokolov, M. M. Apodaca, B. A. Grzybowski, and I. S. Aranson, Swimming Bacteria Power Microscopic Gears, Proc. Natl. Acad. Sci. U.S.A. 107, 969 (2010).

[23] G. Li and J. X. Tang, Accumulation of Microswimmers Near a Surface Mediated by Collision and Rotational Brownian Motion, Phys. Rev. Lett. 103, 078101 (2009).

[24] A. P. Berke, L. Turner, H. C. Berg, and E. Lauga, Hydrodynamic Attraction of Swimming Microorganisms by Surfaces, Phys. Rev. Lett. 101, 038102 (2008).

[25] M. Ramia, D. L. Tullock, and N. Phan-Thien, The Role of Hydrodynamic Interaction in the Locomotion of Microorganisms, Biophys. J. 65, 755 (1993).

[26] H. Shum, E. A. Gaffney, and D. J. Smith, Modelling Bacterial Behaviour Close to a No-Slip Plane Boundary: The Influence of Bacterial Geometry, Proc. R. Soc. A 466, 1725 (2010).

[27] D. Giacché, T. Ishikawa, and T. Yamaguchi, Hydrodynamic Entrapment of Bacteria Swimming Near a Solid Surface, Phys. Rev. E 82, 056309 (2010).

[28] D. Pimponi, M. Chinappi, P. Gualtieri, and C. M. Casciola, Hydrodynamics of Flagellated Microswimmers Near Free-Slip Interfaces, J. Fluid Mech. 789, 514 (2016).

[29] J. Hu, A. Wysocki, R. G. Winkler, and G. Gompper, Physical Sensing of Surface Properties by Microswimmers-Directing Bacterial Motion via Wall Slip, Sci. Rep. 5, 9586 (2015).

[30] T. Eisenstecken, J. Hu, and R. G. Winkler, Bacterial Swarmer Cells in Confinement: A Mesoscale Hydrodynamic Simulation Study, Soft Matter 12, 8316 (2016).

[31] M. A.-S. Vigeant, R. M. Ford, M. Wagner, and L. K. Tamm, Reversible and Irreversible Adhesion of Motile Escherichia Coli Cells Analyzed by Total Internal Reflection Aqueous Fluorescence Microscopy, Appl. Environ. Microbiol. 68, 2794 (2002).

[32] G. Li, L.-K. Tam, and J.X. Tang, Amplified Effect of Brownian Motion in Bacterial Near-Surface Swimming, Proc. Natl. Acad. Sci. U.S.A. 105, 18355 (2008).

[33] M. Molaei, M. Barry, R. Stocker, and J. Sheng, Failed Escape: Solid Surfaces Prevent Tumbling of Escherichia Coli, Phys. Rev. Lett. 113, 068103 (2014).

[34] H. C. Berg, E. Coli in Motion (Springer Science \& Business Media, Berlin, Heidelberg, 2008).

[35] J. Schwarz-Linek, J. Arlt, A. Jepson, A. Dawson, T. Vissers, D. Miroli, T. Pilizota, V. A. Martinez, and W. C. K. Poon, Escherichia Coli as a Model Active Colloid: A Practical Introduction, Colloids Surf., B 137, 2 (2016).

[36] S. Choi, P. Kim, R. Boutilier, M. Y. Kim, Y. J. Lee, and H. Lee, Development of a High Speed Laser Scanning Confocal Microscope with an Acquisition Rate up to 200 Frames per Second, Opt. Express 21, 23611 (2013). 
[37] J. B. Pawley and B. R. Masters, Handbook of Biological Confocal Microscopy, Opt. Eng. 35, 2765 (1996).

[38] F. Saglimbeni, S. Bianchi, A. Lepore, and R. Di Leonardo, Three-Axis Digital Holographic Microscopy for High Speed Volumetric Imaging, Opt. Express 22, 13710 (2014).

[39] S. Bianchi, F. Saglimbeni, A. Lepore, and R. Di Leonardo, Polar Features in the Flagellar Propulsion of E. Coli Bacteria, Phys. Rev. E 91, 062705 (2015).

[40] J. Sheng, E. Malkiel, and J. Katz, Digital Holographic Microscope for Measuring Three-Dimensional Particle Distributions and Motions, Appl. Opt. 45, 3893 (2006).

[41] J. F. Jikeli, L. Alvarez, B. M. Friedrich, L. G. Wilson, R. Pascal, R. Colin, M. Pichlo, A. Rennhack, C. Brenker, and U. Benjamin Kaupp, Sperm Navigation Along Helical Paths in 3D Chemoattractant Landscapes, Nat. Commun. 6, 7985 (2015).

[42] L. G. Wilson, L. M. Carter, and S. E. Reece, High-Speed Holographic Microscopy of Malaria Parasites Reveals Ambidextrous Flagellar Waveforms, Proc. Natl. Acad. Sci. U.S.A. 110, 18769 (2013).

[43] A. Ashkin, J. M. Dziedzic, J. E. Bjorkholm, and S. Chu, Observation of a Single-Beam Gradient Force Optical Trap for Dielectric Particles, Opt. Lett. 11, 288 (1986).

[44] M. Padgett and R. Di Leonardo, Holographic Optical Tweezers and Their Relevance to Lab on Chip Devices, Lab Chip 11, 1196 (2011).

[45] K. C. Neuman, E. H. Chadd, G. F. Liou, K. Bergman, and S. M. Block, Characterization of Photodamage to Escherichia Coli in Optical Traps, Biophys. J. 77, 2856 (1999).

[46] M. B. Rasmussen, L. B. Oddershede, and H. Siegumfeldt, Optical Tweezers Cause Physiological Damage to
Escherichia Coli and Listeria Bacteria, Appl. Environ. Microbiol. 74, 2441 (2008).

[47] T. L. Min, P. J. Mears, L. M. Chubiz, C. V. Rao, I. Golding, and Y. R. Chemla, High-Resolution, Long-Term Characterization of Bacterial Motility Using Optical Tweezers, Nat. Methods 6, 831 (2009).

[48] See Supplemental Material at http://link.aps.org/ supplemental/10.1103/PhysRevX.7.011010 for videos.

[49] E. Lauga, W. R. DiLuzio, G. M. Whitesides, and H. A. Stone, Swimming in Circles: Motion of Bacteria Near Solid Boundaries, Biophys. J. 90, 400 (2006).

[50] O. Sipos, K. Nagy, R. Di Leonardo, and P. Galajda, Hydrodynamic Trapping of Swimming Bacteria by Convex Walls, Phys. Rev. Lett. 114, 258104 (2015).

[51] B. Rodenborn, C.-H. Chen, H. L. Swinney, B. Liu, and H. P. Zhang, Propulsion of Microorganisms by a Helical Flagellum, Proc. Natl. Acad. Sci. U.S.A. 110, E338 (2013).

[52] L. Turner, W. S. Ryu, and H. C. Berg, Real-Time Imaging of Fluorescent Flagellar Filaments, J. Bacteriol. 182, 2793 (2000).

[53] B. Ezhilan and D. Saintillan, Transport of a Dilute Active Suspension in Pressure-Driven Channel Flow, J. Fluid Mech. 777, 482 (2015).

[54] A. J. Wolfe, M. Patricia Conley, T. J. Kramer, and H. C. Berg, Reconstitution of Signaling in Bacterial Chemotaxis, J. Bacteriol. 169, 1878 (1987).

[55] S. Taheri-Araghi, S. Bradde, J. T. Sauls, N. S. Hill, P. Anne Levin, J. Paulsson, M. Vergassola, and S. Jun, Cell-Size Control and Homeostasis in Bacteria, Curr. Biol. 25, 385 (2015). 